\title{
Implantable biomedical sensor array with biocompatible hermetic encapsulation
}

\author{
Carola Jorsch $^{1}$, Ulrike Schmidt ${ }^{1}$, David Ulkoski ${ }^{2}$, Carmen Scholz ${ }^{2}$, Margarita Guenther ${ }^{1}$, and \\ Gerald Gerlach $^{1}$ \\ ${ }^{1}$ Solid-State Electronics Laboratory, Technische Universität Dresden, Dresden, Germany \\ ${ }^{2}$ Department of Chemistry, University of Alabama in Huntsville, Huntsville, AL, USA \\ Correspondence to: Carola Jorsch (carola.jorsch@tu-dresden.de)
}

Received: 21 February 2016 - Revised: 26 May 2016 - Accepted: 8 June 2016 - Published: 6 July 2016

\begin{abstract}
The treatment of metabolic diseases, such as diabetes mellitus, requires sensitive measuring systems. These should be able to detect the different metabolism-related parameters (blood glucose level, $\mathrm{pH}, p \mathrm{CO}_{2}$ ) simultaneously and continuously. A new approach is an implantable wireless sensor microarray consisting of several hydrogel-based piezoresistive sensors that can provide an on-line monitoring of physiological parameters in the human body fluid. The specifically customized stimuli-responsive hydrogels enable the development of reliable biosensors for different analytes. In this regard, the on-line medical diagnostics attracts the main interest. The developed sensor system and its encapsulation should correspond to high requirements on the biocompatibility of implants according to the medical standard DIN EN ISO 10993-5. A multi-layer sensor encapsulation consisting of parylene $\mathrm{C}$ and amphiphilic block copolymers was proposed for subcutaneous implants and characterized using contact angle measurements and X-ray photoelectron spectroscopy. In vitro studies with model cells showed no cytotoxicity of the polyethylene glycol-based block copolymers. In order to understand the behavior of implants under physiological conditions, the interaction of the implant surface with biological specimen like proteins is discussed, taking into account the possible protein adsorption on the implant surface due to tissue inflammation around the implant, which should be minimized. Finally, the biocompatibility of the developed sensor system was studied to prove the suitability of the approach.
\end{abstract}

\section{Introduction}

Treatment of metabolic diseases, e.g., diabetes mellitus, could be improved by continuously monitoring physiological parameters like $\mathrm{pH}$, blood sugar and $p \mathrm{CO}_{2}$ (partial pressure of $\mathrm{CO}_{2}$ ). The studied sensor arrays allow a simultaneous measurement of such control parameters.

Especially for medical applications, a glucose-sensitive material fills a significant need. There are actually different types of concepts to determine the glucose concentration. A widely used principle is the enzyme-linked detection with a combination of glucose oxidase and catalase. However, these sensors can only provide a single value at the time of measurement. However, the glucose level fluctuates throughout the day from lowest glucose level in the morning to a higher level after meals. Correspondingly, it is recommended that type 1 diabetes patients with defects in insulin production pass a self blood glucose monitoring at least three times a day (Sacks et al., 2002). In recent years, continuous monitoring systems based on enzymes have been developed. These are in use but need an improvement concerning their accuracy, calibration, operation period and the difficulties associated with sterilization processes (Egawa et al., 2014).

In a second type of sensor, boronic acid, which reversibly forms a cyclic ester with the cis-1,2- or 1,3-diol structures of sugars, is used as glucose-sensitive unit (Fig. 1). The synthetic chemical ligands are easier to handle, more stable and of low cost compared to enzyme-based systems.

In this work, the boronic acid groups were integrated in a hydrogel network by a polymerization with acrylamide. This network swells or shrinks in dependence on the glucose concentration in a solution and deflects a thin bending plate of a piezoresistive pressure sensor. This enzyme-free principle al- 


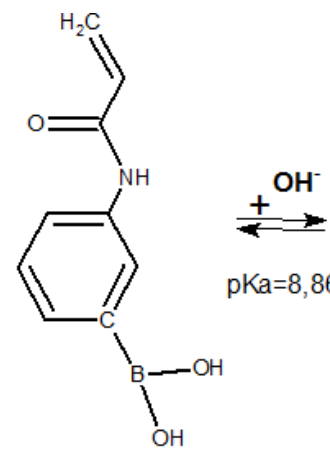

Deswelling form

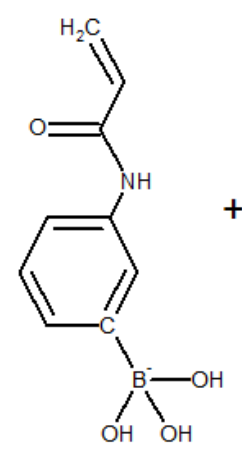

Transition form
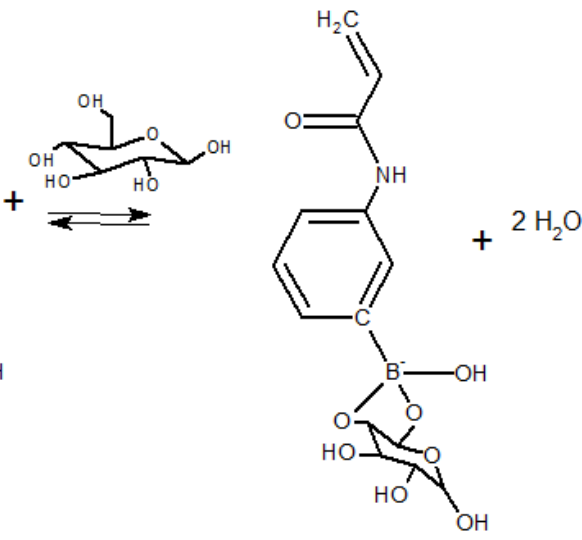

Swelling form

Figure 1. A glucose linkage mechanism of phenylboronic acid acrylamide dependent on the pKa value.

lows a greater variety of synthesis and manufacturing conditions and is less critical to harsh influences like sterilization.

The implemented system consists of a variety of hydrogelbased piezoresistive sensors. The working principle of the sensors as well as the type of the stimuli-responsive hydrogel depends on the applications, such as temperature sensor, $\mathrm{pH}$ sensor, glucose sensor and more (Gerlach and Arndt, 2009; Lin et al., 2010).

The whole sensor system is featured with a biocompatible encapsulation needed for device implantation into the human body. This work concentrates on the polymer-based hermetic encapsulation for medical devices, its properties and its behavior in biological milieus.

\section{Materials and methods}

\subsection{Sensor array}

Figure 2 illustrates the setup of a pressure sensor array as it was used for the experiments of this work. It consists of several piezoresistive pressure sensors for glucose, $\mathrm{pH}$ and $p \mathrm{CO}_{2}$. Each of them has an integrated piezoresistive Wheatstone bridge at the surface of the flexure plate which acts as mechanoelectrical transducer for the transformation of the plate deflection into an electrical output voltage $V_{\text {out }}$ (Guenther et al., 2012, 2014). The aqueous solution to be measured diffuses through the porous hydrophilic aluminum oxide membrane into the chip cavity. The stimuli-responsive hydrogel swells and deflects the bending plate. An increase of $V_{\text {out }}$ corresponds to a hydrogel swelling, whereas a hydrogel shrinking leads to the decrease of $V_{\text {out }}$.

The glucose sensor as well as the $\mathrm{pH}$ sensor have the very same layout. The $p \mathrm{CO}_{2}$ sensor comprises two chambers separated by a porous membrane: the first one with a hydrogel and the second one with a sodium bicarbonate solution. The reservoir with the bicarbonate solution is separated from the aqueous environment by a hydrophobic gas-permeable mem- brane. $\mathrm{CO}_{2}$ permeates through this membrane and leads to $\mathrm{pH}$ changes in the $\mathrm{NaHCO}_{3}$ solution. These alternate values cause the swelling and deswelling of the hydrogel and finally the sensor signal.

\subsection{Hydrogels}

In this sensor array, different hydrogels with special components were used for the $\mathrm{pH}, p \mathrm{CO}_{2}$, temperature and glucose sensing. The cationic polymer N,N-dimethylaminoethyl methacrylate (DMAEMA) is pH-sensitive and essential for the $\mathrm{pH}$-responsive hydrogel. For the gel preparation, N-(2-hydroxypropyl) methacrylamide (HPMA), triethylene glycol dimethacrylate (TEGDMA, as a crosslinker), and DMAEMA were used in a mole ratio 60/40/01 (HPMA/DMAEMA/TEGDMA) (Schmidt et al., 2015).

For the glucose-sensitive hydrogel, 3(acrylamido)phenylboronic acid (APB) and acrylamide (AAM) were mixed with $\mathrm{N}, \mathrm{N}$ '-methylenebisacrylamide (BIS, as a crosslinker) and lithium phenyl-2,4,6trimethylbenzoyl phosphinate (LAP, as a UV initiator) and then irradiated using a UV lamp (UVACUBE 100, Höhnle AG) for a few minutes.

\subsection{Biocompatible encapsulation}

An inert barrier layer of parylene $\mathrm{C}$ was deposited onto the whole device surface except the ceramic membrane out of the vapor phase (First Sensor Microelectronic Packaging GmbH Dresden, Parylene Coating System 2000-150 LV) (Kirsten et al., 2013). All areas of the surface are uniformly coated by the gaseous parylene $\mathrm{C}$.

Afterwards, the hydrophobic and optically transparent parylene $\mathrm{C}$ layer was activated in an oxygen plasma, silanized and then covered with crosslinked polyethylene glycolated (PEG) poly(amino acids), which establish an inherent amphiphilicity gradient (Obeid and Scholz, 2011). 


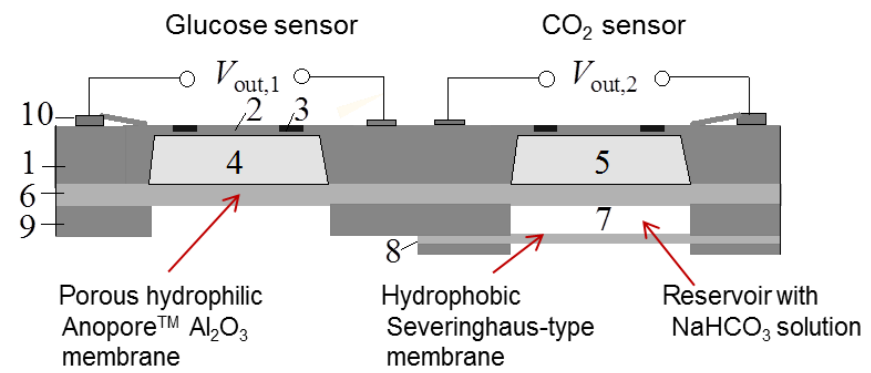

1 Si chip; 2 Bending plate; 3 Mechano-electrical transducer (piezoresistive Wheatstone bridge); 4, 5 Swellable hydrogel; 6 Porous, hydrophilic Anopore ${ }^{\mathrm{TM}} \mathrm{Al}_{2} \mathrm{O}_{3}$ membrane; 7 Reservoir with $\mathrm{NaHCO}_{3}$ solution; 8 Hydrophobic membrane; 9 Si substrate; 10 Interconnect

Figure 2. Schematic setup of a hydrogel-based sensor array comprising a glucose and a $\mathrm{CO}_{2}$ sensor.

The samples coated with an amphiphilic block copolymer were washed three times with ethanol. The polymer coating was investigated by means of contact angle measurements (OCA 20, DataPhysics Instruments) and X-ray photoelectron spectroscopy (XPS, ESCA 5700, Physical Electronics PHI).

\subsection{In vitro experiments}

The cells used for the assessment of the material biocompatibility were human fibroblasts (Coriell Institute, NJ, USA). The cell line was grown as a monolayer culture in the Minimum Essential Medium (MEM) with Earle's salts containing L-glutamine, fetal bovine serum and non-essential amino acids in an atmosphere of $5 \% \mathrm{CO}_{2}$ in the air.

Cells were seeded in 12-well plates with coated samples at a density of $4 \times 10^{4}$ cells per well in $1 \mathrm{~mL}$ of medium. These plates were incubated for $72 \mathrm{~h}$ at $37^{\circ} \mathrm{C}$ and $80 \%$ relative humidity.

Cell viability was measured with a CellTiter-Blue Assay (Promega). After cultivation, $500 \mu \mathrm{L}$ of CellTiter-Blue solution were added to each well. After an incubation period of $4 \mathrm{~h}$, the fluorescence at $560_{\mathrm{Ex}} / 590_{\mathrm{Em}} \mathrm{nm}$ was measured with a microplate reader (Infinite ${ }^{\circledR} 200$ Pro, Tecan).

For an apoptosis detection, the cells were cultivated as described and dyed afterwards with propidium iodide (PI). The cells were incubated for $30 \mathrm{~min}$ in the dark, detached from the surface and washed with a phosphate-buffered saline solution (PBS). Apoptotic, necrotic and vital cells were detected using a FACScan ${ }^{\mathrm{TM}}$ (Becton Dickinson, argon laser $488 \mathrm{~nm})$.

\subsection{Protein adhesion}

The interaction between implantable biomaterials and tissue is mainly influenced by proteins and a cell signalling. The adhesion of proteins like fibronectin promotes the cell signalling and the attachment of cells. Furthermore, it provides the adverse inflammation process.
To study the adhesion behavior of such proteins on different surfaces, a fluorescence-labeled fibronectin HiLyte 488 (BIOMOL) was used. The samples were incubated at $37^{\circ} \mathrm{C}$ for $30 \mathrm{~min}$ in the dark and washed subsequently three times with PBS. The area fluorescence intensity was measured with the help of the microplate reader.

\subsection{IL8 expression}

Interleukin 8 is one of the major mediators of the inflammatory response and secreted by several cell types. Samples were collected over time from the cell culture as described. After reaching $80 \%$ confluence, the supernatant was aspirated and stored at $-20^{\circ} \mathrm{C}$ until an additional use. As a control, a part of the samples were stimulated with lipopolysaccharides (LPS). These endotoxins unleash an inflammatory reaction and increase the IL8 expression of cells.

The assay used to determine the IL8 expression employs the quantitative sandwich enzyme immunoassay technique (Arigo Biolaboratories). An antibody specific for IL8 was pre-coated onto a microtiter plate. Standards and samples were pipetted into the wells, and IL8 was bound to the immobilized antibody. After washing, a biotin-conjugated antibody specific for IL8 was added to each well and incubated. Afterwards, a washing was performed in order to remove unbound substances, then a streptavidin-conjugated horseradish peroxidase (HRP) was added and incubated. Subsequently, any unbound antibody enzyme reagent was washed out and a substrate solution was added. Color developed in proportion to the amount of IL8 bound in the initial step. The concentration of IL8 was determined by comparing the optical density of samples with the standard curve.

\section{Results}

The swelling properties of hydrogels were determined by studying free swelling of $1 \mathrm{~mm}$ thick hydrogels (size $3 \mathrm{~mm} \times 2 \mathrm{~mm}$ ) in PBS with and without glucose. The results of glucose-sensitive hydrogels (AAm/PBA/BIS; 80/20/0.25) 


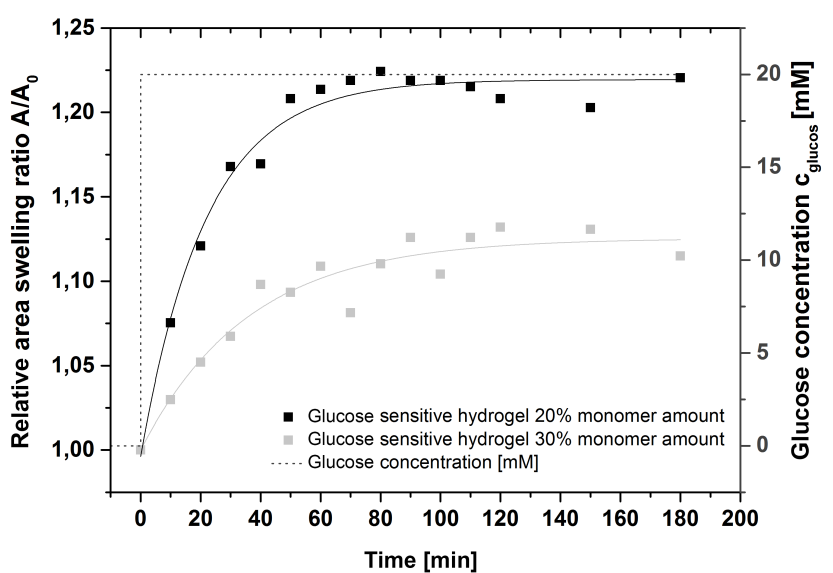

Figure 3. Relative area swelling ratio of the glucose-sensitive hydrogel with different total monomer amounts of 20 and $30 \mathrm{wt} \%$ in PBS with $20 \mathrm{mM}$ glucose and without glucose.

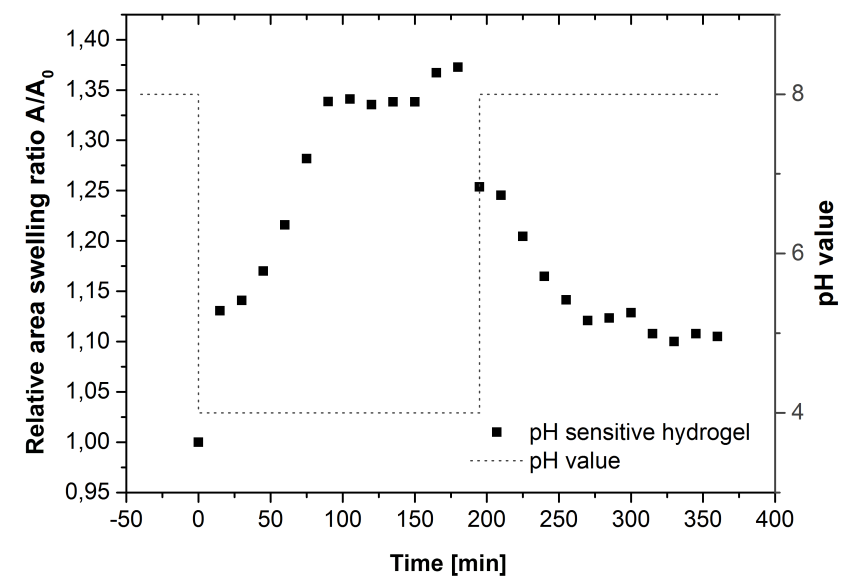

Figure 4. Relative area swelling ratio of $\mathrm{pH}$-sensitive hydrogels in PBS while swelling from $\mathrm{pH} 8$ to $\mathrm{pH} 4$ and reverse deswelling.

with different total monomer amounts (20 and $30 \mathrm{wt} \%$ ) are shown in Fig. 3. One can see the higher swelling ratio of hydrogels with less monomer amount. Also the response time was reduced.

A pH-dependant swelling/shrinking process was observed for the hydrogel synthesized with HPMA/DMAEMA/TEGDMA. Figure 4 shows the swelling ratio in PBS with different $\mathrm{pH}$ values. The relative area swelling ratio of 1.3 is sufficient to deflect the bending plate of the sensor. The first sensor curve is shown in Fig. 5 where the $\mathrm{pH}$ dependent swelling/shrinking of a HPMA/DMAEMA/TEGDMA hydrogel in $17 \mathrm{mM} \mathrm{NaHCO}_{3}$ leads to a deflection of the bending plate. The $\mathrm{pH}$ change in the surrounding solution was induced by $\mathrm{CO}_{2}$ and $\mathrm{N}_{2}$ supply. A fast sensor response showed already after a few minutes the correlation of $p \mathrm{CO}_{2}$ and $\mathrm{pH}$ in $\mathrm{NaHCO}_{3}$ solution as well as the $\mathrm{pH}$-dependent gel response.

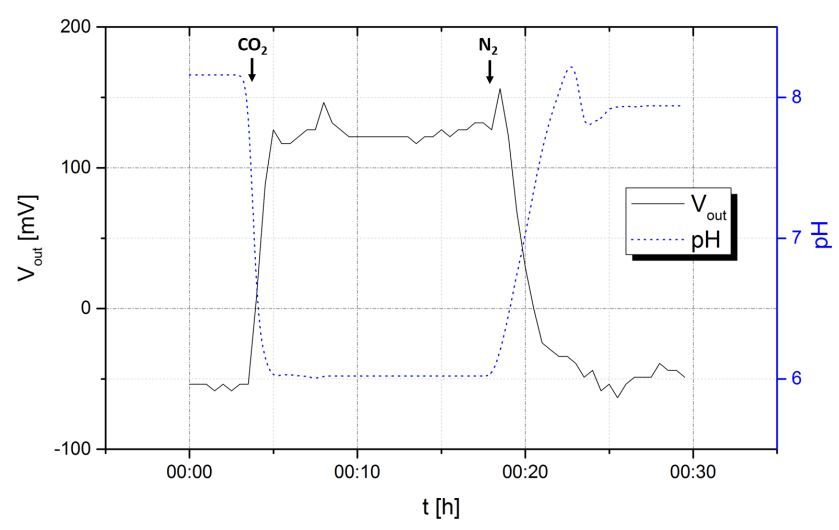

Figure 5. Sensor output voltage during the swelling of a HPMA/DMAEMA/TEGDMA hydrogel in $17 \mathrm{mM} \mathrm{NaHCO}_{3}$ while changing the $\mathrm{pH}$ through $\mathrm{CO}_{2}$ and $\mathrm{N}_{2}$ supply. (a)

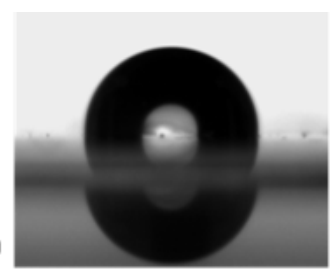

(b)

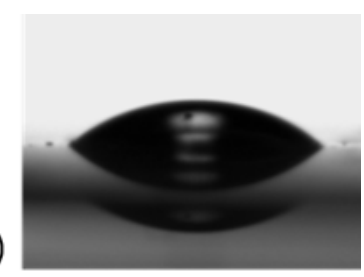

Figure 6. Contact angle measurements with water on (a) parylene C, $\theta=90.0^{\circ}$; (b) parylene C/amphiphilic block copolymer, $\theta=44.3^{\circ}$.

The change of the surface properties during the surface coating process from pure parylene $\mathrm{C}$ to the amphiphilic block copolymer layer on it was detected by the contact angle $\theta$ using the sessile-drop technique (Fig. 6).

The surface properties changed from hydrophobic $(\theta=$ $\left.90.0^{\circ}\right)$ to hydrophilic $\left(\theta=44.3^{\circ}\right)$. Also, a surface free energy $\sigma_{\text {sample }}$, calculated according to Owens-Wendt, changed its value from $16.6 \mathrm{mN} \mathrm{m}^{-1}$ for the untreated parylene $\mathrm{C}$ to $49.42 \mathrm{mN} \mathrm{m}^{-1}$ for the parylene C/amphiphilic block copolymer-coated sample. These changes may have various effects on the cell and protein adhesion in a physiological environment.

The XPS experimental data of parylene $\mathrm{C}$ and the amphiphilic block copolymer coated sample are compared in Table 1. Prior to measuring, the samples were sputtered with $4 \mathrm{keV}$ argon ions to clean the surface from moisture and contaminations. The coating composition was evaluated by calculating the peak areas of high-resolution XP spectra by means of the PHI MultiPak software.

One can see the changes in the oxygen, carbon and nitrogen content due to the different chemical compositions of parylene $\mathrm{C}$ and the amphiphilic block copolymer of PEG and poly(amino acids) (benzyl-L-glutamate (L-glu) and Lleucine (L-leu)) (Fig. 7). The amount of silicon after the coating relies on the prior silanization of the parylene $\mathrm{C}$ surface. 
Table 1. Experimental data of XPS measurements.

\begin{tabular}{llllll}
\hline \multirow{2}{*}{ Material } & \multicolumn{5}{c}{ Concentration (at \%) } \\
\cline { 2 - 6 } & $\mathrm{C} 1 \mathrm{~s}$ & $\mathrm{~N} 1 \mathrm{~s}$ & $\mathrm{O} 1 \mathrm{~s}$ & $\mathrm{Si} 2 \mathrm{p}$ & $\mathrm{C} 12 \mathrm{p}$ \\
\hline Parylene C & 96.77 & & 0.62 & & 2.61 \\
\hline Block copolymer & 77.49 & 2.73 & 14.4 & 3.39 & 2.01 \\
\hline
\end{tabular}

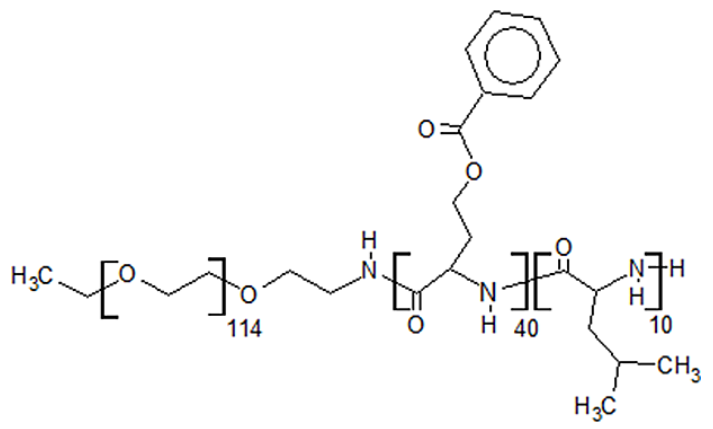

Figure 7. Structural formula of the block copolymer $\mathrm{PEG}_{114}$-bp(L-Glu) 40 -b-p(L-Leu) 10 .

One can imply that these changes in composition indicate the successful coating of the inert parylene $\mathrm{C}$ surface.

The effects of the materials on cell growth and cell viability were most significant for parylene $\mathrm{C}$. The cell viability decreased to $60 \%$ for parylene C (Fig. 8), while the samples with the block copolymer-coated surface showed only a $20 \%$ drop in viability compared to the control (a cell culture well material). This means that the block copolymer-coated surface is non-toxic according to the DIN EN ISO 109935:2009 for medical products. The block copolymer-coating demonstrated a better biocompatibility compared to the pure parylene $\mathrm{C}$.

Figure 9 shows the results of PI staining for the detection of apoptotic, necrotic and vital cells. A higher amount of apoptotic cells on the parylene $\mathrm{C}$ surface confirms the results of the cell viability assay. The influence of block copolymer on the cell adhesion is normal and displays the biocompatibility of the material.

The adhesion behavior of fibronectin is summarized in Fig. 10. The fluorescence intensity on the control surface was much higher than on the block copolymer-coated material. The hydrophobic parylene $\mathrm{C}$ layer shows higher protein adhesion compared to the block copolymer-coated material. The adhesion of fibronectin can be decreased by about $50 \%$ due to the block copolymer coating.

The immunological experiments with the cytokine IL8 showed no significant higher expression of IL8. Only the stimulated control material displayed the regular IL8 concentration after an inflammatory reaction (Fig. 11).

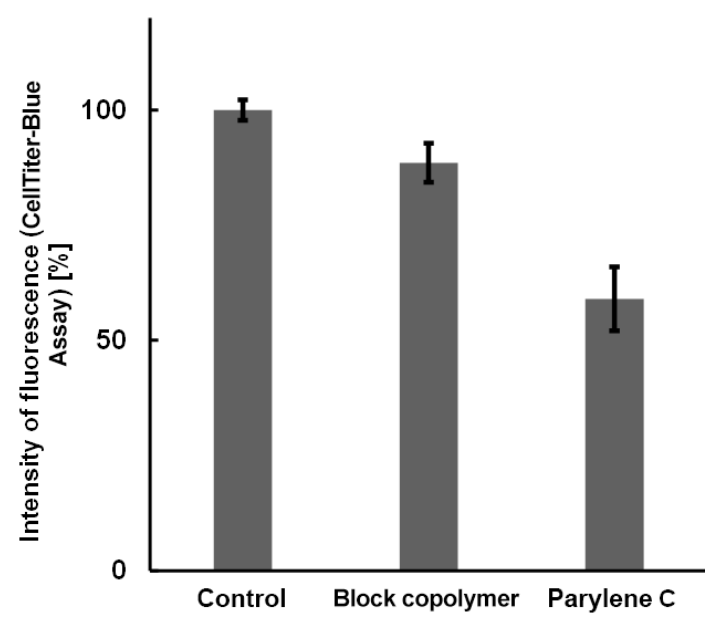

Figure 8. Fluorescence intensity (CellTiter-Blue Assay) as an indicator for the cell viability after the direct incubation on the coated biomaterials $(n=6)$.

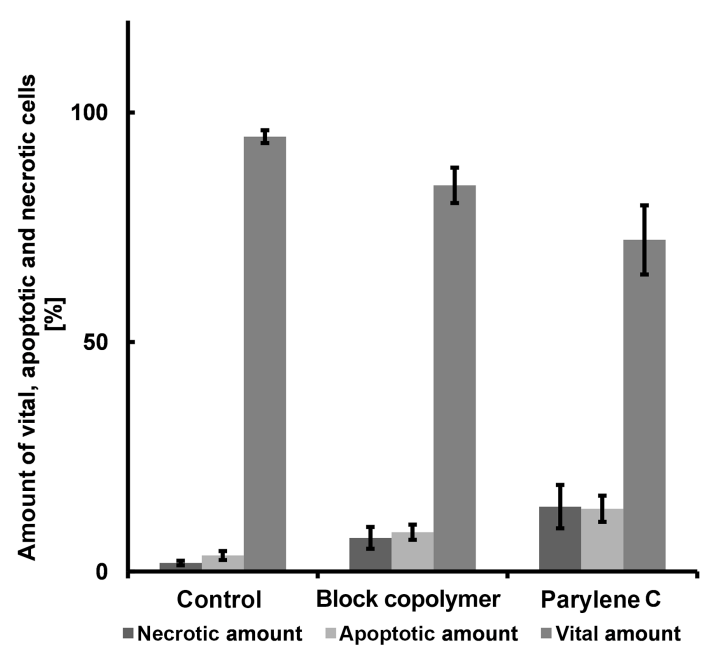

Figure 9. Vital, apoptotic and necrotic amount of cells after PI staining detected by means of the FACScan $(n=6)$.

\section{Discussion}

The investigated swelling degrees of glucose-sensitive hydrogels show a dependence on the total monomer amount. With less total monomer, the hydrogel response is stronger to glucose but the hydrogel material is more brittle and not easy to handle.

A hydrogel with $\mathrm{pH}$-sensitive groups was shown during free swelling. The $\mathrm{pH}$ variances from $\mathrm{pH} 4$ to $\mathrm{pH} 8$ are conducive to relative area swelling ratios of ca. 1.3. Our further study will be concentrated on the $\mathrm{pH}$ values in a physiological range near $\mathrm{pH}$ 7.4.

In the sensors targeted, a thinner (200-300 $\mu \mathrm{m})$ hydrogel layer was used than for the free-swelling measurements. In general, the thinner the hydrogel layers are, the faster they 


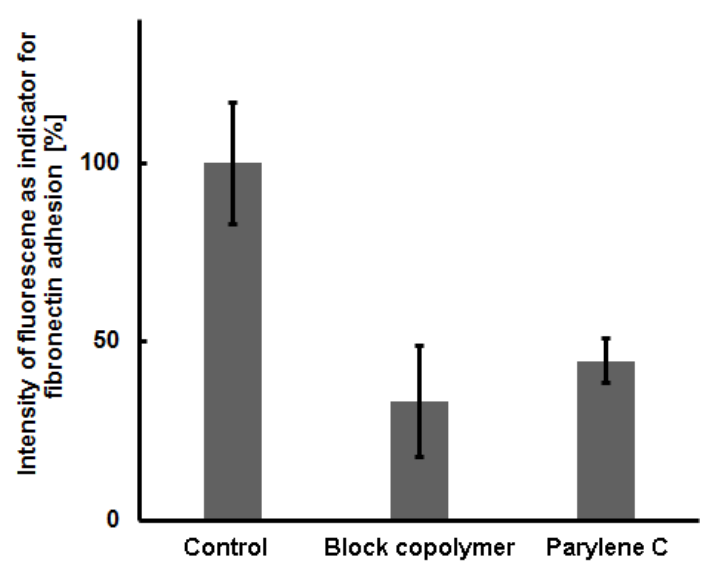

Figure 10. Intensity of fluorescence as an indicator for fibronectin adhesion after incubation with labeled fibronectin HiLyte 488 ( $n=$ 5).

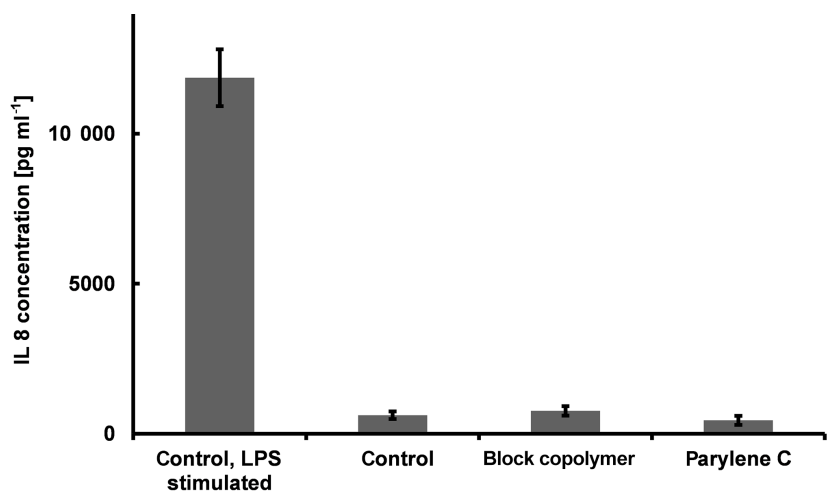

Figure 11. IL8 expression of human fibroblasts after the incubation on parylene $\mathrm{C}$, on the block copolymer as well as on the control materials $(n=6)$.

swell. As a consequence, the response time of the sensor was decreased, as shown in Fig. 5.

The multilayer encapsulation influences the surface characteristics of implantable biosensors. The surface coating, either with parylene $\mathrm{C}$ or with the amphiphilic block copolymer change, the surface contact angle of water and the surface free energy. These parameters play a major role in the protein and cell adhesion. The hydrophobic parylene $\mathrm{C}$ surface shows a cell-repellent behavior. The more hydrophilic surface with a higher surface energy leads to a better biocompatibility. More experiments are needed to prove this result. For this purpose, the study of the time-dependent adhesion, the adhesion strength and of the focal adhesion contacts can be used. The fibroblasts used in this work represent the examples of the types of cells which the implants will be in contact with during a clinical use.

It is difficult to functionalize the inert parylene $\mathrm{C}$. The coupling of the amphiphilic block copolymer was accomplished through physical adhesion after the treatment of the parylene
C surface in oxygen plasma. This could be improved by covalent coupling techniques.

In general, the developed multilayer coating should show a protein-like behavior. The poly(amino acid) segments of the block copolymer mimic a protein layer and the PEG segments exert a stealth character. The decrease of immune response is a positive consequence of this special property.

This effect correlates strongly with the cell viability and with the normal apoptosis rate after the incubation on the materials. The non-toxic characteristic supports the proteinrepulsive and non-inflammatory behavior.

In summary, the multilayer encapsulation not only protects the electronic components from the environmental fluids but also provides a good surface biocompatibility. This is especially important for the implants of all types. Many different applications could benefit from such multilayer encapsulations.

\section{Conclusions}

A sensor array with different stimuli-sensitive hydrogels for implantable biosensors has been developed. The swelling ratios of UV-crosslinked hydrogels were determined which are sufficient for sensor applications. The response time has still to be improved.

In order to develop a biocompatible and hermetic encapsulation, a multi-layer concept was used. Parylene C, as a hermetic barrier layer, was deposited first via chemical vapor deposition (CVD). After oxygen plasma activation and silanization, the amphiphilic block copolymer adheres to the surface. To further improve the adhesion, a covalent coupling of additional block copolymers will be investigated in the future.

Different characteristics, like changes in a layer composition and hydrophilicity, were shown with XPS and contact angle measurements.

The biocompatibility was demonstrated with cell experiments using human fibroblasts as a model for the subcutaneous application. Cell viability, apoptosis rate, necrosis rate, protein adhesion and immune response on coated samples were studied. The polymer-coated material is non-toxic and exhibits a protein-repellent behavior which strongly decreases an immune response after the implantation.

Acknowledgements. The authors thank Sergej Zigler from First Sensor Microelectronic Packaging GmbH for the parylene C coating, the biomedical laboratory of the Fraunhofer Institute for Organic Electronics, Electron Beam and Plasma Technology (FEP) for the assistance with the cell experiments, and the Deutschen Forschungsgemeinschaft (DFG) for the financial support (Research Training Group 1865).

Edited by: T. Scheper

Reviewed by: two anonymous referees 


\section{References}

Egawa, Y., Miki, R., and Seki, T.: Colorimetric sugar sensing using boronic acid-substituted azobenzenes, Materials, 7, 1201-1220, 2014.

Gerlach, G. and Arndt, K.-F. (Hrsg.): Hydrogel Sensors and Actuators. Engineering and Technology. Berlin, Heidelberg, SpringerVerlag, 2009.

Guenther, M., Gerlach, G., Wallmersperger, T., Avula, M. N., Cho, S. H., Xie, X., Devener, B. V., Solzbacher, F., Tathireddy, P., Magda, J. J., Scholz, C., Obeid, R., and Armstrong, T.: Smart hydrogel-based biochemical microsensor array for medical diagnostics, Adv. Sci. Tech., 85, 47-52, 2012.

Guenther M., Wallmersperger T., and Gerlach G.: Piezoresistive chemical sensors based on functionalized hydrogels, Chemosensors, 2, 145-170, 2014.

Kirsten, S., Wetterling, J., Uhlemann, J., Wolter, K., and Zigler, S.: Barrier properties of polymer encapsulation materials for implantable microsystems, in: Electronics and Nanotechnology (ELNANO), 2013 IEEE XXXIII International Scientific Conference, 269-272, 2013.

Lin, G., Chang, S., Hao, H., Tathireddy, P., Orthner, M., Magda, J., and Solzbacher, F.: Osmotic swelling pressure response of smart hydrogels suitable for chronically implantable glucose sensors, Sensor. Actuat. B-Chem., 144, 332-336, 2010.

Obeid, R. and Scholz, C.: Synthesis and self-assembly of welldefined poly(amino acid) end-capped poly(ethylene glycol) and poly(2-methyl-2-oxazoline), Biomacromolecules, 12, 37973804, 2011.

Sacks, D. B., Bruns, D. E., Goldstein, D. E., Maclaren, N. K., McDonald, J. M., and Parrott, M.: Guidelines and recommendations for laboratory analysis in the diagnosis and management of diabetes mellitus, Clin. Chem., 48, 436-472, 2002.

Schmidt, U., Günther, M., and Gerlach, G. (Hrsg.): Biochemische piezoresistive Sensorkonzepte für die Biotechnologie und medizinische Anwendungen. Forschungsgesellschaft für Messtechnik, Sensorik und Medizintechnik e.V. Dresden; AMA Verband für Sensorik und Messtechnik e.V., Berlin, 2015. 